BULL. AUSTRAL. MATH. SOC.

VOL. 29 (1984), 19-34.

\title{
A TRANSLATION PLANE OF ORDER 81 AND ITS FULL COLLINEATION GROUP
}

\author{
Vito Abatangelo
}

\begin{abstract}
In this paper a new translation plane of order 81 is constructed. Its collineation group is solvable and acts on the line at infinity as a permutation group $K$ which is the product of a group of order 5 belonging to the center of $K$ with a group of order 48 . A 2-Sylow subgroup of $K$ is the direct product of a dihedral group of order 8 with a group of order 2 . $K$ admits six orbits. They have lengths $4,6,12,12,24$, 24.
\end{abstract}

\section{Introduction}

Let $G F(q)$ be a Galois field of odd order $q(\geq 5)$ and let $F$ be the regular spread of $\mathrm{PG}(3, q)$. Suppose that $F$ contains a set $R$ of reguli satisfying the properties

(i) $R$ consists of $(q+3) / 2$ reguli, $R_{1}, R_{2}, \ldots, R_{(q+3) / 2}$,

(ii) any two reguli of $R$ have exactly two lines in common,

(iii) no three reguli of $R$ have a line in common.

Received 2 September 1983. Research partially supported by MPI (Research project "Structture Geometriche Combinatorie e Loro Applicazioni"). The author wishes to thank G. Korchmaros for the discussion with him about the argument of the present paper.

Copyright Clearance Centre, Inc. Serial-fee code: 0004-9727/84 $\$ A 2.00+0.00$. 
Let $U$ be the partial spread consisting of all the lines of the reguli $R_{i}(i=1,2, \ldots,(q+3) / 2)$ and let $I_{R}$ denote the point set covered by $U$. If $R_{i}^{\prime}$ denotes the opposite regulus of $R_{i}$, the set $U^{\prime}$ of all the lines of $R_{i}^{\prime}$ is not a spread of $I_{R}$ because any point $P$ of $I_{R}$ belongs to two lines of $U^{\prime}$. According to a result of Bruen [4], if a subset $V$ of $U^{\prime}$ is a partial spread then $V$ contains $(q+1) / 2$ lines of each $R_{i}^{\prime}$ such that the union of these half-reguli is $V$. If there exists such a partial spread $V$ then one can obtain a new spread $G=(F-U) \cup V$ by replacing $U$ with $V . G$ will be called $\beta$-derived from $F$ (with respect to $(U, V)$ ). We can try to obtain a new partial spread $V=\frac{1}{2} U^{\prime}$ of $I_{R}$ by choosing only a suitable half of the lines of each opposite regulus. As for $\beta$-derived spreads the existence problem is yet unsolved except for $q=5,7,11,13$. Specific examples are given in Bruen's paper [4] when $q=5,7$, in [5] and [12] when $q=11$ and in [13] when $q=13$.

In [4] Bruen also proved that if $q \geq 7$ the collineation group of a B-derived spread is the inherited group, that is, it is the subgroup of the collineation group of $\mathrm{PG}(3, q)$ which leaves $F$ invariant. The collineation groups of the translation planes arising from the above mentioned examples are determined [5], [12], [13].

In this paper the first example of a $\beta$-derived spread is constructed for $q=9$ and the collineation group of the corresponding translation plane is determined. It is a solvable group satisfying the properties listed in the summary.

\section{Notation and terminology}

Notation and terminology are the same as in Bruen's paper [4]. The reader is assumed to be familiar with the theory of projective planes given in Dembowski [6] and in Hughes and Piper [7] and with the theory of permutation groups given in Wielandt [14].

Let $G F(9)$ be the quadratic extension of $G F(3)=\{0,1,2\}$. In particular let us consider the polynomial $x^{2}+x+2$ (which is irreducible in $\mathrm{GF}(3)), i \notin \mathrm{GF}(3)$ such that $i^{2}+i+2=0$ and 
$3=i, 4=i+1,5=i+2,6=2 i, 7=1+2 i, 8=2+2 i$; so $\mathrm{GF}(9)=\{0,1,2,3,4,5,6,7,8\}$.

The homogeneous coordinates $(x, y, z, u)$ denote points in $\operatorname{PG}(3, q)$. The line joining the point $\left(x_{1}, y_{1}, z_{1}, u_{1}\right)$ to the point $\left(x_{2}, y_{2}, z_{2}, u_{2}\right)$ is denoted by $\left\langle\left(x_{1}, y_{1}, z_{1}, u_{1}\right),\left(x_{2}, y_{2}, z_{2}, u_{2}\right)\right\rangle$; moreover if $g, h, \ldots, w$ are elements of a group, then the subgroup generated by $g, h, \ldots, w$ is denoted by $(g, h, \ldots, w)$.

\section{Preliminaries}

Before the example, the general relation between the spread $F$ and the Miquelian inversive plane $M(q)$ over $G F(q)$ is discussed briefly.

Assume that $q$ is odd and denote by $s$ a non-square element of $\mathrm{GF}(q)$, so $\mathrm{GF}\left(q^{2}\right)=\left\{a+b t \mid a, b \in \mathrm{GF}(q), t^{2}=s\right\}$. We define norm $N(a+b t)$ of $a+b t$ of $\mathrm{GF}\left(q^{2}\right), N(a+b t)=a^{2}-s b^{2}$. Clearly $N(a+b t) \in \mathrm{GF}(q)$; moreover set $N(\rho)=\{a+b t \mid N(a+b t)=\rho\} . M(q)$ is considered the incidence structure whose points are the elements of $\mathrm{GF}\left(q^{2}\right) \cup\{\infty\}$ and whose circles are the subsets of $\mathrm{GF}\left(q^{2}\right) \cup\{\infty\}$ of the following types :

(I) $a+b t+N(\rho)=\{x+y t \mid N((a-x)+(b-y) t\}=\rho\}$;

(II) $\quad c(a+b t, c+d t)=\{(a+b t)+\lambda(c+d t) \mid \lambda \in G F(q) \cup\{\infty\}\}$.

The spread $F$ is the union of all the lines

$$
\langle(1,0,0,0),(0,1,0,0)\rangle,\langle(a, s b, 0,1),(b, a, 1,0)\rangle
$$

where $a, b$ run over $G F(q)$. By a theorem of Bruck's ([3], also [4]), the map

$$
\begin{aligned}
\infty & \rightarrow((1,0,0,0),(0,1,0,0)) \\
\psi: \quad & a+b t \rightarrow(\{a, 6 b, 0,1),(b, a, 1,0))
\end{aligned}
$$

is an incidence preserving isomorphism between $M(q)$, with its points and circles, and $F$, with its lines and reguli.

By Bruck's theorem, the problem of finding sets $R$ of reguli satisfying the properties (i), (ii), (iii) is equivalent to that of constructing chains $C$ of circles in $M(q)$ satisfying the properties 
(i)' $C$ consists of $(q+3) / 2$ circles, $c_{1}, c_{2}, \ldots, c_{(q+3) / 2}$,

(ii)' any two circles of $\mathcal{C}$ have exactly two points in common,

(iii)' no three circles of $\mathcal{C}$ have a point in common.

Let $W$ be the collineation group of $P G(3, q)$ mapping $F$ onto itself. Every $w \in W$ determines a map $w^{\prime}$ of $M(q)$ onto itself defined as follows: let $P$ be any point of $M(q)$ and let $r$ be the line of $F$ which corresponds to $P$ by $\Psi$; then $w^{\prime}(P)$ is the point which corresponds to $w\left(x^{2}\right)$ by $\Psi$. Therefore a map $\Phi$ is defined putting $\Phi(w)=w^{\prime}$. Of course $\Phi$ is a homomorphism of $W$ into $W^{\prime}$, where $W^{\prime}$ denotes the automorphism group of $M(q)$. The kernel of $\Phi$ is the group $M$ of all collineations of $\mathrm{PG}(3, q)$ fixing $F$ linewise. $M$ is a cyclic group of order $q+1$. Moreover $W \simeq \Gamma L\left(2, q^{2}\right)$ and $W^{\prime} \simeq \operatorname{P\Gamma L}\left(2, q^{2}\right)$.

Let $H$ be the subgroup of $W$ which leaves invariant a set $R$ of reguli satisfying the properties (i), (ii), (iii). If $H^{\prime}$ denotes the subgroup of $W^{\prime}$ which leaves invariant the chain $C$ of circles of $M(q)$ corresponding to $\mathrm{R}$ by $\Psi$, then $\Phi(H)=H^{\prime}$. Hence $H / M \simeq H^{\prime}$.

\section{Two partial spreads $U$ and $V$ of $P G(3,9)$ covering the same point set}

Using the notation of Bruen's paper, our construction can be described as follows.

$$
\begin{aligned}
& \text { If } s=3, \text { the norm-classes of } G F(9) \text { are } \\
& N(0)=\{0\}, \\
& N(1)=\{1,2,4+4 t, 8+4 t, 3+5 t, 6+5 t, 3+7 t, 6+7 t, 4+8 t, 8+8 t\}, \\
& N(2)=\{5,7,4+t, 8+t, 4+2 t, 8+2 t, 3+3 t, 6+3 t, 3+6 t, 6+6 t\}, \\
& N(3)=\{5+3 t, 7+3 t, 3+4 t, 6+4 t, 5 t, 5+6 t, 7+6 t, 7 t, 3+8 t, 6+8 t\}, \\
& N(4)=\{3+t, 6+t, 3+2 t, 6+2 t, 3 t, 1+5 t, 2+5 t, 6 t, 1+7 t, 2+7 t\}, \\
& N(5)=\{4,8,1+3 t, 2+3 t, 5+5 t, 7+5 t, 1+6 t, 2+6 t, 5+7 t, 7+7 t\}, \\
& N(6)=\{t, 2 t, 4+3 t, 8+3 t, 1+4 t, 2+4 t, 4+6 t, 8+6 t, 1+8 t, 2+8 t\}, \\
& N(7)=\{3,6,1+t, 2+t, 1+2 t, 2+2 t, 5+4 t, 7+4 t, 5+8 t, 7+8 t\}, \\
& N(8)=\{5+t, 7+t, 5+2 t, 7+2 t, 4 t, 4+5 t, 8+5 t, 4+7 t, 8+7 t, 8 t\} .
\end{aligned}
$$

An easy calculation shows that the following circles of $M(q)$ form a chain $C$ satisfying the properties (i)', (ii)', (iii)': 


$$
\begin{aligned}
& c_{1}: 2 t+N(7), C_{2}: t+N(7), c_{3}: 7 t+N(5), \\
& c_{4}{ }^{*}: 5 t+N(5), c_{5}:(0,3+t), c_{6}:(0,3+2 t) .
\end{aligned}
$$

The corresponding reguli $R_{1}, R_{2}, R_{3}, R_{4}, R_{5}, R_{6}$ contained in $F$ form a set $R$ satisfying the properties (i), (ii), (iii). The line $\langle(1,0,0,0),(0,1,0,0)\rangle$ is denoted by $r_{\infty}$ and the line $\langle(a, 3 b, 0,1),(b, a, 1,0)\rangle$ by $r(a, b)$, then

$$
\begin{aligned}
& R_{1}=\{r(1,0), r(2,0), r(1,1), r(2,1), r(3,2) \text {, } \\
& r(6,2), r(5,3), r(7,3), r(5,7), r(7,7)\} \text {, } \\
& R_{2}=\{r(1,0), r(2,0), r(3,1), r(6,1), r(1,2) \text {, } \\
& r(2,2), r(5,5), r(7,5), r(5,6), r(7,6)\}, \\
& R_{3}=\{r(5,0), r(7,0), r(1,1), r(2,1), r(1,4) \text {, } \\
& r(2,4), r(5,5), r(7,5), r(4,7), r(8,7)\} \text {, } \\
& R_{4}=\{r(5,0), r(7,0), r(1,2), r(2,2), r(4,5), \\
& r(8,5), r(5,7), r(7,7), r(1,8), r(2,8)\}, \\
& R_{5}=(r(0,0), r(3,1), r(6,2), r(7,3), r(1,4), \\
& \left.r(4,5), r(5,6), r(8,7), r(2,8), r_{\infty}\right\}, \\
& R_{6}=\{r(0,0), r(6,1), r(3,2), r(5,3), r(2,4) \text {, } \\
& \left.r(8,5), r(7,6), r(4,7), r(1,8), r_{\infty}\right\} \text {. }
\end{aligned}
$$

Therefore

$$
\begin{aligned}
U= & \{r(1,0), r(2,0), r(1,1), r(2,1), r(3,2), \\
& r(6,2), r(5,3), r(7,3), r(5,7), r(7,7), r(3,1), r(6,1), r(1,2), \\
& r(2,2), r(5,5), r(7,5), r(5,6), r(7,6), r(5,0), r(7,0), r(1,4), \\
& r(2,4), r(4,7), r(8,7), r(4,5), r(8,5), \\
& \left.\quad r(1,8), r(2,8), r(0,0), r_{\infty}\right\} .
\end{aligned}
$$

Denote by $V$ the union of the following half-reguli

$$
\frac{1}{2} R_{i}^{\prime}=\left\{r^{\prime}(i, 1), r^{\prime}(i, 2), r^{\prime}(i, 3), r^{\prime}(i, 4), r^{\prime}(i, 5)\right\}:
$$$$
\frac{1}{2} R_{1}^{\prime}=\{((2,3,0,1),(0,1,1,0)\rangle,\langle(2,0,0,1),(1,1,1,0)\rangle,
$$$$
\langle(6,6,0,1),(2,3,1,0)\rangle,\langle(7,8,0,1),(3,5,1,0)),
$$$$
((7,7,0,1),(7,5,1,0))\},
$$ 


$$
\begin{array}{r}
\frac{1}{2} R_{2}^{\prime}=\{(1,0,0,1),(2,2,1,0)\},\langle(1,6,0,1),(0,2,1,0)\}, \\
((3,3,0,1),(1,6,1,0)\},((5,5,0,1),(5,7,1,0)\}, \\
\quad(5,4,0,1),(6,7,1,0)\}\},
\end{array}
$$

$\frac{1}{2} R_{3}^{\prime}=\{(1,1,0,1),(1,2,1,0)\},\langle(1,3,0,1),(4,2,1,0))$,

$$
\begin{aligned}
((7,4,0,1),(0,5,1,0)) & ((7,0,0,1),(5,5,1,0)), \\
& ((8,8,0,1),(7,4,1,0))\},
\end{aligned}
$$$$
\frac{1}{2} R_{4}^{\prime}=\{(2,6,0,1),(8,1,1,0)\},\langle(2,2,0,1),(2,1,1,0)\},
$$$$
((4,4,0,1),(5,8,1,0)),((5,0,0,1),(7,7,1,0)\} \text {, }
$$$$
((5,8,0,1),(0,7,1,0))\} \text {, }
$$

$$
\begin{array}{r}
\frac{1}{2} R_{5}^{\prime}=\{\langle(4,1,0,0),(0,0,1,0)\},\langle(7,1,0,0),(0,0,1,1)), \\
((1,0,0,0),(0,0,1,2)),((8,1,0,0),(0,0,1,3)\}, \\
\\
\quad(5,1,0,0),(0,0,1,6)\rangle\},
\end{array}
$$

$$
\begin{array}{r}
\frac{1}{2} R_{6}^{\prime}=\{((0,1,0,0),(0,0,1,4)),\langle(6,1,0,0),(0,0,1,5)\}, \\
((1,1,0,0),(0,0,1,7)),((3,1,0,0),(0,0,1,8)\}, \\
\\
\quad(2,1,0,0),(0,0,0,1)\rangle\} .
\end{array}
$$

Note that $\frac{1}{2} R_{i}^{\prime}$ is contained in the opposite regulus $R_{i}^{\prime}$ of $R_{i}$. An explicit calculation shows that $U$ and $V$ cover the same subset $I_{R}$ of $\mathrm{PG}(3,9)$ and have no lines in common. Therefore $G=(F-U) \cup V$ is a spread of $\mathrm{PG}(3,9)$. Clearly $G$ is a $B$-derived spread from $F$.

\section{The automorphism group of the chain $\mathcal{C}$}

In this section the automorphism group $H^{\prime}$ of $M(9)$ which leaves the point set $I$ covered by $C$ invariant is determined. As a model of $M(9)$ we will take the geometry $G(Q)$ of the plane sections of an elliptic quadric $Q$ of $\operatorname{PG}(3,9)$. The passage from $M(9)$ to $G(Q)$ can be realized as follows. Let $Q$ be the elliptic quadric of $\operatorname{PG}(3,9)$ whose equation is

$$
Q: z u=x^{2}+6 y^{2} .
$$

As it is well known, the map

$$
\begin{aligned}
\sigma: \quad & \rightarrow(0,0,1,0) \\
a+b t & \rightarrow\left(a, b, a^{2}+6 b^{2}, 1\right)
\end{aligned}
$$


is an incidence preserving isomorphism between $M(9)$ and $Q$ with their points and circles. Moreover ( $c f .[6], p .274)$, if $w^{\prime}$ is an automorphism of $M(9)$, then there is a unique collineation $\bar{w}$ of $\operatorname{PG}(3,9)$ leaving $Q$ invariant such that $\omega^{\prime}$ acts on $M(9)$ as $\bar{w}$ on $Q$. Thus

$$
\sigma\left(\omega^{\prime}(P)\right)=\bar{w}(\sigma(P)) \text { for every } P \in M(9)
$$

and

$$
\sigma^{-1}(\bar{\omega}(P))=\omega^{\prime}\left(\sigma^{-1}(P)\right) \text { for every } P \in Q .
$$

Therefore the problem of determining the automorphism group $H^{\prime}$ of $M(9)$ which leaves $I$ invariant is equivalent to that of determining the collineation group $\bar{H}$ of $\mathrm{PG}(3,9)$ which maps $Q$ onto itself and leaves $\sigma(I)$ invariant, where $\sigma(I)=\{\sigma(P) \mid P \in I\}$. First some lemmas are needed.

LEMMA 5.1. Let $D$ be a circle of $Q$ contained in $\sigma(I)$; then $D$ coincides with a circle $\sigma\left(C_{i}\right)$.

Proof. By way of contradiction, let $D$ be distinct from any circle $\sigma\left(C_{i}\right)$; then for every $i=1,2, \ldots, 6$ we have $\left|D \cap \sigma\left(C_{i}\right)\right| \leq 2$ and from this

$$
\sum_{i=1}^{6}\left|D \cap \sigma\left(c_{i}\right)\right| \leq 2 \cdot 6=12 .
$$

On the other hand each point of $D$ lies exactly on two circles $\sigma\left(c_{i}\right)$, so

$$
\sum_{i=1}^{6}\left|D \cap \sigma\left(C_{i}\right)\right|=2 \cdot 10=20 .
$$

Therefore we would have $20 \leq 12$.

LEMMA 5.2. Let $Z$ be a collineation group of $\mathrm{PG}(3,9)$ which maps $Q$ onto itself leaving $\sigma(I)$ invariant; then $Z$ preserves the chain $\sigma(C)$.

Proof. Let $\alpha \in Z$ and $\sigma\left(C_{i}\right) \in \sigma(C)$; as $\alpha\left(\sigma\left(C_{i}\right)\right)$ is a circle contained in $\sigma(I)$, the preceding Lemma 5.1 assures that $\alpha\left(\sigma\left(C_{i}\right)\right)$ is a circle of $\sigma(C)$. This proves Lerma 5.2 . 
Let $\pi_{i}(i=1,2, \ldots, 6)$ be the plane of $\operatorname{PG}(3,9)$ which meets $Q$ in $\sigma\left(c_{i}\right)$. Denote by $P_{i}$ the polo of $\pi_{i}$ with respect to $Q$. Then, by Lemma 5.2, $\bar{H}$ is the collineation group of $\operatorname{PG}(3,9)$ which maps $Q$ onto itself and leaves the set $\left\{P_{1}, P_{2}, P_{3}, P_{4}, P_{5}, P_{6}\right\}$ invariant. An easy calculation shows that $P_{1}(0,2,2,1), P_{2}(0,1,2,1), P_{3}(0,7,1,1)$, $P_{4}(0,5,1,1), P_{5}(1,1,0,0), P_{6}(2,1,0,0)$.

LEMMA 5.3. $\bar{H}$ fixes $Y_{\infty}$.

Proof. The lines joining $P_{1}$ and $P_{2}, P_{3}$ and $P_{4}, P_{5}$ and $P_{6}$ pass through $Y_{\infty}$; there exist only three planes joining $Y_{\infty}$ and four points among $P_{i}(i=1,2, \ldots, 6)$. So $\bar{H}$ must fix $Y_{\infty}$.

Let $\bar{H}_{i}$ be the stabilizer of $P_{i}$. First we note that

LEMMA 5.4. $\bar{H}_{1}=\bar{H}_{2}, \bar{H}_{3}=\bar{H}_{4}, \bar{H}_{5}=\bar{H}_{6}$.

LEMMA 5.5. The identity collineation $I$ is the only collineation of $\bar{H}$ fixing each $P_{i}(i=1,2, \ldots, 6)$.

Proof. The only non-identity collineation which leaves each $P_{i}$ invariant has equations

$$
\rho x^{\prime}=x^{3}, \rho y^{\prime}=y^{3}, \rho z^{\prime}=2 u^{3}, \rho u^{\prime}=2 z^{3} ;
$$

but this collineation does not preserve $Q$.

LEMMA 5.6. $\bar{H}$ acts faithfully on $\left\{P_{1}, P_{2}, \ldots, P_{6}\right\}$.

LEMMA $5.7 . \bar{H}_{6}$ is a dihedral group of order $8 \cdot \bar{H}_{6}$ is generated by the following collineations:

$$
\begin{aligned}
& \bar{h}: \rho x^{\prime}=5 x, \rho y^{\prime}=5 y, \rho z^{\prime}=2 z, \rho u^{\prime}=u, \\
& \bar{s}: \rho x^{\prime}=x, \quad \rho y^{\prime}=y, \quad \rho z^{\prime}=u, \quad \rho u^{\prime}=z \text {. }
\end{aligned}
$$

$\bar{h}$ and $\bar{s}$ act on $\left\{P_{1}, P_{2}, \ldots, P_{6}\right\}$ as folzows:

$$
\bar{h}:\left(P_{1} P_{3} P_{2} P_{4}\right)\left(P_{5}\right)\left(P_{6}\right) \text { and } \bar{s}:\left(P_{1} P_{2}\right)\left(P_{3}\right)\left(P_{4}\right)\left(P_{5}\right)\left(P_{6}\right) \text {. }
$$

Proof. By Lemmas 5.4 and 5.6 we have that $\bar{H}_{6}$ is a subgroup of the 
symetric group $S_{4}$ on four objects. An easy calculation shows that $\bar{h}$ and $\bar{s}$ act on $\left\{P_{1}, P_{2}, \ldots, P_{6}\right\}$ as said before and $\bar{h}^{4}=\bar{s}^{2}=(\bar{h} \bar{s})^{2}=1$. So $\langle\bar{h}, \bar{s}\rangle$ is a dihedral group of order 8 contained in $\bar{H}_{6}$. As $\bar{H}_{1}=\bar{H}_{2}$ we have $\bar{H}_{1} \cap \bar{H}_{6}=\bar{H}_{2} \cap \bar{H}_{6}$ and so $\bar{H}_{6}$ cannot contain any element of order 3 . Thus $\left|\bar{H}_{6}\right| \leq 8$ and therefore $\bar{H}_{6}=\langle\bar{h}, \bar{s}\rangle$.

As $\left|\bar{H}_{6}\right|=8$ and the orbit of $P_{6}$ has length at most 6 , the following lemma holds.

LEMMA 5.8. $|\bar{H}| \leq 48$.

Let us consider the collineation

$\bar{c}: \rho x^{\prime}=5 z+7 u, \rho y^{\prime}=5 y, \rho z^{\prime}=x+z+u, \rho u^{\prime}=x+2 z+2 u$.

An easy calculation shows

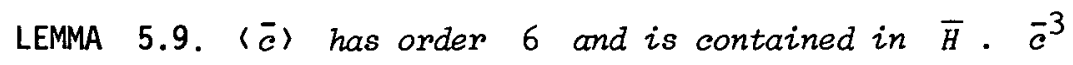
centralizes $\vec{H}_{6}$.

Next we prove

LEMMA 5.10. $(\bar{c}) \cap \bar{H}_{6}=\{1\}$.

Proof. By Lemma 5.9, $\left|\langle\bar{c}\rangle \cap \bar{H}_{6}\right| \leq 2$. If $\left|\langle\bar{c}\rangle \cap \bar{H}_{6}\right|=2$, then $\bar{c}^{3} \in \bar{H}_{6}$ and, by Lemma $5.7, \bar{c}^{-3}=\bar{h}^{2}$, as $\bar{h}^{2}$ is the central involution of $\bar{H}_{6}$. But one can verify that $\bar{c}^{3} \neq \bar{h}^{2}$.

THEOREM 5.11. $\bar{H}=\langle\bar{h}, \bar{s}, \bar{c}\rangle,|\bar{H}|=48$ and a 2-Sylow subgroup of $\bar{H}$ is the direct product of a dihedral group of order 8 with a group of order 2 .

Proof. By Lemmas $5.7,5.10,\langle\bar{h}, \bar{s}, \bar{c}\rangle$ is a subgroup of order at least 48 of $\bar{H}$. By Lemma $5.8,|\bar{H}| \leq 48$, thus $\bar{H}=\langle\bar{h}, \overline{8}, \bar{c}\rangle$ and $|\bar{H}|=48$. The latter assertion of the theorem follows from Lemmas 5.7 and 5.9 .

\section{The collineation group $H$ preserving $U$}

Let us denote by 1 the identity collineation of $\mathrm{PG}(3,9)$ and put 
(6.1) $m_{\alpha}: \rho x^{\prime}=\alpha x+y, \rho y^{\prime}=3 x+\alpha y, \rho z^{\prime}=\alpha z+3 y, \rho u^{\prime}=z+\alpha u$, where $\alpha \in G F(9)$. Then

LEMMA 6.1. The cyclic group of order 10 ,

$$
M=\left\{m_{\alpha} \mid \alpha \in G F(9)\right\} \cup\{1\},
$$

fixes $U$ Zinewise; moreover $M=\left\langle m_{3}\right\rangle \cdot M$ is the full collineation group of $\mathrm{PG}(3,9)$ which fixes $U$ linewise.

Lemmas 6.2 and 6.3 follow from Lemma 6.1 .

LEMMA 6.2. $M$ is the subgroup of $H$ fixing each regulus $R_{i}$. Therefore $H / M$ acts on $\left\{R_{1}, R_{2}, \ldots, R_{6}\right\}$ faithfulzy.

Proof. Let $\Phi$ be the correspondence defined in Section 3. Then $\Phi(H)=\bar{H}$ and $\Phi(H)$ acts on $\left\{R_{1}, R_{2}, \ldots, R_{6}\right\}$ as $\bar{H}$ on $\left\{P_{1}, P_{2}, \ldots, P_{6}\right\}$. By Lerma $6.6, \bar{H}$ is faithful on $\left\{P_{1}, P_{2}, \ldots, P_{6}\right\}$. Thus Ker $\Phi$ is the subgroup of $H$ fixing each $R_{i}$. As we have shown in Section 3, Ker $\Phi=M$. This proves Lemma 6.2.

Since $|\bar{H}|=48$ and $|M|=10$, from Lemma 6.2 it follows that

LEMMA 6.3. $|H|=480$.

Let us consider the collineations

(6.2) $h: \rho x^{\prime}=5 x$,

$\rho y^{\prime}=5 y$,

$\rho z^{\prime}=z$, $\rho u^{\prime}=u$,

(6.3) $s: \rho x^{\prime}=z$,

$\rho y^{\prime}=6 u$,

$\rho z^{\prime}=6 x$, $p u^{\prime}=y$,

(6.4) $c: \rho x^{\prime}=5 y+7 z, \rho y^{\prime}=8 x+4 u, \rho z^{\prime}=3 x+3 u, \rho u^{\prime}=2 y+2 z$.

It is easy to check that $h, s, c$ have order $4,2,6$ respectively and

$$
\left\{\begin{array}{l}
h\left(r_{\infty}\right)=r_{\infty}, \\
h(r(a, b))=r(5 a, 5 b),
\end{array}\right.
$$


(6.6) $\left\{\begin{array}{l}s\left(r_{\infty}\right)=r(0,0), \\ s(r(0,0))=r_{\infty}, \\ s(r(a, b))=r\left(a\left(a^{2}+6 b^{2}\right)^{-1}, b\left(a^{2}+6 b^{2}\right)^{-1}\right) \text { for }(a, b) \neq(0,0),\end{array}\right.$

$$
\left\{\begin{array}{l}
c\left(r_{\infty}\right)=r(7,0), \\
c(r(2,0))=r_{\infty}, \\
c(r(a, b))=r\left(\left(7 a^{2}+4 b^{2}+5\right)\left((a+1)^{2}+6 b^{2}\right)^{-1}, 7 b\left((a+1)^{2}+6 b^{2}\right)^{-1}\right) \\
\text { for }(a, b) \neq(2,0) .
\end{array}\right.
$$

Therefore

LEMMA 6.4. $(h, s, c, M\rangle$ is a subgroup of $H$.

From $(6.5),(6.6)$ and $(6.7)$ we infer that $h, s, c$ act on $\left\{R_{1}, R_{2}, \ldots, R_{6}\right\}$ as follows:

$$
\begin{aligned}
& h:\left(R_{1} R_{3} R_{2} R_{4}\right)\left(R_{5}\right)\left(R_{6}\right), s:\left(R_{1} R_{2}\right)\left(R_{3}\right)\left(R_{4}\right)\left(R_{5}\right)\left(R_{6}\right), \\
& c:\left(R_{1} R_{6} R_{3} R_{2} R_{5} R_{4}\right) .
\end{aligned}
$$

Thus $h, s, c$ act on $\left\{R_{1}, R_{2}, \ldots, R_{6}\right\}$ as $\bar{h}, \bar{s}, \bar{c}$ on $\left\{P_{1}, P_{2}, \ldots, P_{6}\right\}$. As the subgroup $(\bar{h}, \bar{s}, \bar{c})$ of $S_{6}$ gives a faithful representation of $\bar{H}$ on $\left\{P_{1}, P_{2}, \ldots, P_{6}\right\}$, we get $(h, s, c, M) / M=(\bar{h}, \bar{s}, \bar{c})$. Therefore from Lemma 6.3 it follows that

LEMMA 6.5. $H=(h, s, c, M)$.

Next we prove

LEMMA 6.6. $\langle h, s, c\rangle \cap M=\{1\}$.

Proof. The orbit of $X_{\infty}$ in $\langle h, s, c\rangle$ consists of the points $X_{\infty}$, $y_{\infty}, z_{\infty}, 0,(1,0,0,1),(0,1,1,0),(2,0,0,1),(0,2,1,0)$, $(5,0,0,1),(0,5,1,0),(7,0,0,1),(0,7,1,0)$. The collineation $m_{\alpha}$ maps $x_{\infty}$ into $(\alpha, 3,0,0)$, so $m_{\alpha} \neq\langle h, s, c)$ if $\alpha \neq 0$. Now let us consider $m_{0}$ : it maps $(1,1,0,0)$ into $(4,1,0,0)$; the orbit of $(1,1,0,0)$ in $\langle h, s, c\rangle$ consists of the points $(1,1,0,0)$, $(0,0,1,1),(1,1,1,1),(8,1,0,0),(0,0,6,1),(2,2,1,1)$, $(5,5,1,1),(7,7,1,1),(1,6,6,1),(2,3,6,1),(7,4,6,1)$, $(5,8,6,1)$. Therefore $m_{0} \notin(h, s, c)$. 
From the preceding lemmas we obtain

THEOREM 6.7. The collineation group $H$ of $\mathrm{PG}(3,9)$ which maps $F$ into itself and leaves $U$ invariant is $\langle h, s, c\rangle \cdot M$. Moreover $\langle h, s, c\rangle \simeq \bar{H} \quad(c f$. Theorem 5.11) and $(h, s, c\rangle$ acts on $\left\{R_{1}, R_{2}, \ldots, R_{6}\right\}$ as $\bar{H}$ on $\left\{P_{1}, P_{2}, \ldots, P_{6}\right\}$.

\section{The inherited group $K$}

Bruen [4] proved that if $q \geq 7$ the collineation group $K$ preserving the partial spread $G=(F-U) \cup V$ is the inherited group, that is, the subgroup of $H$ fixing $G$. In our case $K$ is a proper subgroup of $H$. In fact one can check that $m_{3}$ does not preserve $V$. On the other hand $h, s, c, m_{3}^{2} \in K$, as

$$
\begin{aligned}
h: & \left(r^{\prime}(1,1) r^{\prime}(3,3) r^{\prime}(2,2) r^{\prime}(4,5)\right)\left(r^{\prime}(1,2) r^{\prime}(3,4) r^{\prime}(2,1) r^{\prime}(4,4)\right) \\
& \left(r^{\prime}(1,3) r^{\prime}(3,5) r^{\prime}(2,3) r^{\prime}(4,3)\right)\left(r^{\prime}(1,4) r^{\prime}(3,2) r^{\prime}(2,5) r^{\prime}(4,1)\right) \\
& \left(r^{\prime}(1,5) r^{\prime}(3,1) r^{\prime}(2,4) r^{\prime}(4,2)\right)\left(r^{\prime}(5,1)\right)\left(r^{\prime}(5,2)\right)\left(r^{\prime}(5,3)\right) \\
& \left(r^{\prime}(5,4)\right)\left(r^{\prime}(5,5)\right)\left(r^{\prime}(6,1)\right) \\
s:= & \left(r^{\prime}(1,1) r^{\prime}(2,1)\right)\left(r^{\prime}(1,2) r^{\prime}(2,2)\right)\left(r^{\prime}(1,3) r^{\prime}(2,3)\right) \\
& \left(r^{\prime}(1,4) r^{\prime}(2,4)\right)\left(r^{\prime}(1,5) r^{\prime}(2,5)\right)\left(r^{\prime}(3,1) r^{\prime}(3,2)\right) \\
& \left(r^{\prime}(3,3) r^{\prime}(3,4)\right)\left(r^{\prime}(3,5)\right)\left(r^{\prime}(4,1) r^{\prime}(4,2)\right)\left(r^{\prime}(4,3)\right) \\
& \left(r^{\prime}(4,4) r^{\prime}(4,5)\right)\left(r^{\prime}(5,1) r^{\prime}(5,3)\right)\left(r^{\prime}(5,2) r^{\prime}(5,4)\right)\left(r^{\prime}(5,5)\right) \\
& \left(r^{\prime}(6,1) r^{\prime}(6,5)\right)\left(r^{\prime}(6,2)\right)\left(r^{\prime}(6,3) r^{\prime}(6,4)\right), \\
c: & \left(r^{\prime}(1,1) r^{\prime}(6,5) r^{\prime}(3,3) r^{\prime}(2,1) r^{\prime}(5,1) r^{\prime}(4,4)\right) \\
& \left(r^{\prime}(1,2) r^{\prime}(6,1) r^{\prime}(3,4) r^{\prime}(2,2) r^{\prime}(5,3) r^{\prime}(4,5)\right) \\
& \left(r^{\prime}(1,3) r^{\prime}(6,2) r^{\prime}(3,5) r^{\prime}(2,3) r^{\prime}(5,5) r^{\prime}(4,3)\right) \\
& \left(r^{\prime}(1,4) r^{\prime}(6,4) r^{\prime}(3,2) r^{\prime}(2,4) r^{\prime}(5,2) r^{\prime}(4,2)\right) \\
& \left(r^{\prime}(1,5) r^{\prime}(6,3) r^{\prime}(3,1) r^{\prime}(2,5) r^{\prime}(5,4) r^{\prime}(4,1)\right),
\end{aligned}
$$

$m_{3}^{2}:\left(r^{\prime}(1,1) r^{\prime}(1,3) r^{\prime}(1,2) r^{\prime}(1,4) r^{\prime}(1,5)\right)$$$
\left(r^{\prime}(2,1) r^{\prime}(2,5) r^{\prime}(2,4) r^{\prime}(2,2) r^{\prime}(2,3)\right)
$$$$
\left(r^{\prime}(3,1) r^{\prime}(3,3) r^{\prime}(3,5) r^{\prime}(3,4) r^{\prime}(3,2)\right)
$$$$
\left(r^{\prime}(4,1) r^{\prime}(4,2) r^{\prime}(4,5) r^{\prime}(4,3) r^{\prime}(4,4)\right)
$$$$
\left(r^{\prime}(5,1) r^{\prime}(5,5) r^{\prime}(5,3) r^{\prime}(5,2) r^{\prime}(5,4)\right)
$$$$
\left(r^{\prime}(6,1) r^{\prime}(6,2) r^{\prime}(6,5) r^{\prime}(6,3) r^{\prime}(6,4)\right) \text {. }
$$ 
Therefore we can state the following:

LEMMA 7.1. $\langle h, s, c\rangle \leq K, m_{3} \notin K, m_{3}^{2} \in K$.

Thus $\left\langle h, s, c, m_{3}^{2}\right\rangle$ is a subgroup of $K$. Since $K \cap M=\left\langle m_{3}^{2}\right\rangle$, $\left|\left\langle m_{3}^{2}\right\rangle\right|=|M| / 2$, it follows that $[H: K]=2$. By $K \neq H$, $K=\left\langle h, s, c, m_{3}^{2}\right\rangle$. Moreover $\left\langle m_{3}^{2}\right\rangle$ is a normal subgroup of $K$. So we have the following:

THEOREM 7.2. The collineation group $K$ preserving the spread $G=(F-U) \cup V$ is the semidirect product of a group of order 5 lying in the center of $K$ with a group isomorphic to $\bar{H}$. Therefore $K$ is a solvable group of order 24 . Moreover the orbits of $K$ are

$$
\begin{aligned}
& (r(3,0) r(4,0) r(6,0) r(8,0)), \\
& (r(0,1) r(0,2) r(0,3) r(0,4) r(0,5) r(0,6) r(0,7) r(0,8) r(3,3) \\
& r(3,5) r(3,6) r(3,7) r(4,1) r(4,2) r(4,4) r(4,8) r(6,3) r(6.5) \\
& r(6,6) r(6,7) r(8,1) r(8,2) r(8,4) r(8,8)), \\
& (r(1,3) r(1,5) r(1,6) r(1,7) r(2,3) r(2,5) r(2,6) r(2,7) r(3,4) \\
& r(3,8) r(4,3) r(4,6) r(5,1) r(5,2) r(5,4) r(5,8) r(6,4) r(6,8) \\
& r(7,1) r(7,2) r(7,4) r(7,8) r(8,3) r(8,6)), \\
& \left(r^{\prime}(1,1) r^{\prime}(1,2) r^{\prime}(2,1) r^{\prime}(2,2) r^{\prime}(3,3) r^{\prime}(3,4) r^{\prime}(4,4) r^{\prime}(4,5)\right. \\
& \left.r^{\prime}(5,1) r^{\prime}(5,3) r^{\prime}(6,1) r^{\prime}(6,5)\right), \\
& \left(r^{\prime}(1,3) r^{\prime}(2,3) r^{\prime}(3,5) r^{\prime}(4,3) r^{\prime}(5,5) r^{\prime}(6,2)\right), \\
& \left(r^{\prime}(1,4) r^{\prime}(1,5) r^{\prime}(2,4) r^{\prime}(2,5) r^{\prime}(3,1) r^{\prime}(3,2) r^{\prime}(4,1) r^{\prime}(4,2)\right. \\
& \left.r^{\prime}(5,2) r^{\prime}(5,4) r^{\prime}(6,3) r^{\prime}(6,4)\right) .
\end{aligned}
$$

\section{The translation complement}

As it is well known a translation plane $\tau$ of order $q^{2}$ arises from any spread of $P G(3, q)$ (cf. [6], p. 220 and [11] and [2]). Here we give an outline for the case $q=9$.

The points of $\tau$ are those of $\operatorname{PG}(4,9)-\operatorname{PG}(3,9)$, the lines of $\tau$ are the planes of $P G(4,9)$ which meet $P G(3,9)$ in a line of the spread and do not belong to $\operatorname{PG}(3,9)$. If we consider $\operatorname{PG}(4,9)-\operatorname{PG}(3,9)$ as a 
vector space $V_{4}(9)$ of dimension 4 over $G F(9)$, the points of $\tau$ can be identified with the vectors of $V_{4}(9)$, the lines through the zero vector 0 are some 2-dimensional subspaces (called also components), the other lines of $\tau$ are translates of the components. The group of collineations of $\tau$ fixing 0 is called the "translation complement" and consists of some linear or semilinear transformations of $v_{4}(9)$. In particular such a transformation $\alpha$ of $V_{4}(9)$ belongs to the translation complement of $\tau$ if and only if the collineation of $\operatorname{PG}(3,9)$ defined by the matrix of $\alpha$ leaves the spread invariant. Moreover if we denote the translation complement of $\tau$ by $\Lambda_{0}$, the cyclic group of order 8 of all dilations with center 0 by $\Delta_{0}$ and the collineation group of $P G(3,9)$ which leaves the spread invariant by $K$, then $K \simeq \Lambda_{0} / \Delta_{0}$.

As $\tau$ is the translation plane arising from the spread $G=(E-U) \cup V$, by Theorem 7.2 we get

THEOREM 8.1. The translation complement $\Lambda_{0}$ of $\tau$ is $(\lambda, \sigma, \gamma, \mu)$, where

$$
\begin{aligned}
& \lambda: x^{\prime}=5 x, \quad y^{\prime}=5 y, \quad z^{\prime}=z, \quad u^{\prime}=u, \\
& \text { o: } x^{\prime}=z, \quad y^{\prime}=6 u, \quad z^{\prime}=6 x, \quad u^{\prime}=y \text {, } \\
& \gamma: x^{\prime}=5 y+7 z, y^{\prime}=8 x+4 u, z^{\prime}=3 x+3 u, u^{\prime}=2 y+2 z, \\
& \mu: x^{\prime}=8 x+y, y^{\prime}=3 x+8 y, z^{\prime}=8 z+3 u, u^{\prime}=z+8 u \text {. }
\end{aligned}
$$

As $\langle\mu\rangle$ is a normal subgroup of $\Lambda_{0}$ and $(\lambda, \sigma, \gamma) \cap(\mu)=\{1\}$, $\Lambda_{0}$ is semidirect product of $(\lambda, \sigma, \gamma)$ and $(\mu)$. Therefore $\Lambda_{0}$ is a solvable group of order $240 \cdot 8=1920$.

\section{References}

[1] L.M. Abatangelo and Vito Abatangelo, "On Bruen's plane of order 25 ", submitted. 
[2] Adriano Barlotti, "Representation and construction of projective planes and other geometric structures from projective spaces", Jber. Deutsch. Math.-Verein. 77 (1975), 28-38.

[3] R.H. Bruck, "Construction problems in finite projective spaces", Constmuction problems in finite projective spaces (C.I.M.E., II Ciclo, Bressanone, 1972. Edizioni Cremonese, Rome, 1973).

[4] Aiden A. Bruen, "Inversive geometry and some new translation planes, I", Geom. Dedicata 7 (1978), 81-98.

[5] M. Capursi, "A translation plane of order 112 ", J. Combin. Theory Ser. A 35 (1983), 289-300.

[6] P. Dembowski, Finite geometries (Ergebnisse der Mathematik und ihrer Grenzgebiete, 44. Springer-Verlag, Berlin, Heidelberg, New York, 1968.

[7] Daniel R. Hughes and Fred C. Piper, Projective planes (Graduate Texts in Mathematics, 6. Springer-Verlag, New York, Heidelberg, Berlin, 1973).

[8] Gábor Korchmáros, "The full collineation group of Bruen's plane of order 49 ", submitted.

[9] Gábor Korchmáros, "A translation plane of order 49 with nonsolvable collineation group", submitted.

[10] B. Larato and G. Raguso, "Il gruppo delle collineazioni di un piano di ordine $13^{2}$ ", Atti del Convegno di Geometria Combinatoria $e$ di Incidenza, 7 (Passo della Mendola, Trento, Italia, 1982. Rendiconti del Seminario Matematico, Brescia, to appear).

[11] T.G. Ostrom, Finite translation planes (Lecture Notes in Mathematics, 158. Springer-Verlag, Berlin, Heidelberg, New York, 1970).

[12] Giuseppe Pellegrino and Gabor Korchmaros, "Translation planes of order $\mathrm{II}^{2} "$, Combinatorial and geometric stmuctures and their applications, Trento, 1980, 249-264 (North-Holland Mathematical Studies, 63. North-Holland, Amsterdam, 1982).

[13] G. Raguso, "Un piano di traslazione di ordine $13^{2}$ ", submitted. 
[14] Helmut Wielandt, Finite permutation groups (translated by R. Bercov. Academic Press, New York, London, 1964).

Dipartimento di Matematica,

Via Re David 200,

70125 Bari,

italy. 